\title{
Effects of rewarding, aversive, and neutral intracranial stimulation administered during flooding (response prevention) in rats*
}

\author{
ROBERT LECLERC and JACQUES ST-LAURENT \\ Département de Psychiatrie, Centre Hospitalier Universitaire \\ Université de Sherbrooke, Sherbrooke, Quebec, Canada \\ and \\ MORRIE BAUM \\ Université de Moncton, Moncton, Nouveau Brunswick, Canada
}

\begin{abstract}
Flooding or response prevention consisted of blocking the learned avoidance response while forcing the animal to stay in the presence of the feared object. The present study investigated the effects of rewarding (in the anterior lateral hypothalamus), aversive (in the dorsomedial tegmentum), and neutral forced intracranial stimulation (ICS) of anterior brain structures during flooding in rats. No effect on avoidance extinction was produced by any type of ICS, while a significant increase of freezing behavior was found during flooding in Ss receiving aversive ICS. These results were discussed in relation to our previous study, which showed that rewarding (in the posterior lateral hypothalamus) and aversive (in the reticularis pontis caudalis) ICS of posterior areas during flooding produced a significant decrease and a tendency to increase avoidance behavior, respectively. It was suggested that the stimulation of posterior areas was more reinforcing (positively and negatively) than the stimulation of anterior structures.
\end{abstract}

Flooding or response prevention consists of preventing the animal Ss from performing a previously learned avoidance response in the presence of the feared stimulus, generally for a protracted period of time. It has been shown that flooding is effective in the elimination of fear-motivated avoidance behavior in animals (Baum, 1966), while its clinical analogue, implosive therapy, produces similar results in the elimination of specific phobias in man (Kirchner \& Hogan, 1966; Hogan \& Kirchner, 1967). Different parameters, such as duration of the flooding period, intensity of the shock trauma used in the avoidance acquisition period, etc. (Baum, 1970), can influence the outcome of the flooding procedure. Various techniques have been shown to make flooding more effective. The introduction of nonfearful rats (social facilitation, Baum, 1969) and, in another experiment, the introduction of a mechanical device forcing the fearful animal to explore (mechanical facilitation, Lederhendler \& Baum, 1970) have been found to enhance the efficacy of flooding in producing avoidance extinction. In other experiments, intracranial stimulation (ICS) in the posterior-lateral hypothalamus

\footnotetext{
*This work was supported by a National Research Council of Canada grant to Dr. M. Baum and by Medical Research Council of Canada Grant MA 3289 and an establishment grant from the Conseil de la Recherche Médicale du Quebec, both to Dr. J. St-Laurent. Thanks are expressed to Marcel Paradis, TD, for skillful technical assistance and to Mary Mitchell for criticisms. Requests for reprints should be sent to Robert Leclerc, Département de Psychologie, Université de Moncton, Moncton, Nouveau Brunswick, Canada. A part of this paper has been submitted for presentation at the ACFAS Convention in Ottawa, October 1972.
}

(PLH) known to originate self-stimulation (Olds, 1958) and to induce "approach behavior," i.e., exploration, an increase in motor activity, sniffing, searching in animals (St-Laurent \& Beaugrand, 1972), has been applied to fearful rats during flooding, and in these rats a rapid cessation of avoidance behavior has subsequently been observed (Gordon \& Baum, 1971; Baum, Leclerc, \& St-Laurent, in press). On the other hand, ICS in the ventral part of the reticularis pontis caudalis (RPC), known to induce intense "escape behavior," i.e., squealing and/or jumping and/or retreating (St-Laurent \& Beaugrand, 1972), had a tendency to increase the number of responses in avoidance extinction when administered during flooding, in comparison with a control group which did not receive any stimulation (Baum, Leclerc, \& St-Laurent, in press). This last experiment suggested that the type of behavior induced during flooding by stimulation of particular brain structures is of great importance in relation to the facilitative action of flooding on avoidance extinction.

In a previous observation study of ICS on the topographic organization of approach behavior (as measured by self-stimulation rate) and escape behavior in rats, St-Laurent and Beaugrand (1972) suggested the existence of a gradient of self-stimulation and escape reactions along the course of the medial forebrain bundle (MFB) and of a more dorsal pathway [amygdala, septum, medial hypothalamus, dorsal portion of the medial tegmentum (DMT), RPC]. They observed that the stimulation of the most posterior areas yielded the highest rate of self-stimulation (e.g., in the PLH) or extremely intense escape behavior (e.g., in the RPC), while the stimulation of the more anterior structures induced lower rate of self-stimulation [e.g., in the 
anterior lateral hypothalamus (ALH)] or less-intense escape behavior (e.g., in the DMT). In order to give further evaluation of St-Laurent and Beaugrand's suggestion, the present experiment investigated the effects on avoidance extinction of ICS during flooding of anterior brain structures, namely, the ALH and the DMT.

\section{METHOD}

The animals consisted of 50 hooded female rats of the Long-Evans origin, obtained from the Canadian Breeding Laboratory, St. Constant, Quebec. They were maintained with food and water always available and, at the time of avoidance training, weighed $175-250 \mathrm{~g}$.

The avoidance apparatus used was entirely automated and was the same as that described extensively in previous studies (Baum, 1966). Briefly, it consisted of a large plywood and Plexiglas box fitted with a grid floor through which scrambled electric shock could be administered. The shock generator was commercially made (by Grason-Stadler) and the intensity was set at 1.3 microamps at all times. Into one side of the cube-shaped box projected a $2 \frac{1}{2}$-in.-wide safety ledge. The rat could escape or avoid shock by jumping or climbing onto the ledge, where its presence was detected by a photocell system. The safety ledge was automatically retractable (via an electric motor device) and a quick, momentary retraction of the ledge resulted in the rat's falling to the grid floor; no discrete warning signal was employed.

The equipment used in surgery and to provide the ICS, as well as the eight single-lever Skinner boxes (the latter made by Scientific Prototype) used to screen animals for self-stimulation, were those used previously by St-Laurent and Beaugrand (1972). The intensity of ICS employed for both the aversive and rewarding electrode placements was always 150 microamps (peak to peak, measured via an oscilloscope, from a 60-cps sine-wave source) and the duration of each pulse was always $0.25 \mathrm{sec}$. The indwelling electrodes used were bipolar and made by winding $1 / 100$-in. (in diam) stainless-steel wire insulated except at the crosstip. Twisting of leads connecting stimulator to rat brain was prevented by use of a commutator-swivel system.

Five groups were used in the study: Group $\mathrm{S}^{+}(\mathrm{N}=10), \mathrm{S}^{-}$ $(N=10), S^{n}(N=10)$, NS $(N=10)$, and TC, a time control group $(N=10)$. All groups were chronically implanted with electrodes in their brains. Positive (rewarding), negative (aversive), or "neutral" (neither rewarding nor aversive) ICS was given to Groups $\mathrm{S}^{+}, \mathrm{S}^{-}$, and $\mathrm{S}^{n}$, respectively, during flooding, while no ICS was given to Group NS. Group TC, a time control group, did not receive any flooding or ICS.

Animals were anesthetized with pentobarbital $(50 \mathrm{mg} / \mathrm{kg}$, IP). Surgical procedures followed those described by Olds and Milner (1954). The bipolar stainless-steel electrodes were aimed stereotaxically to lodge the exposed tip in the anterior lateral hypothalamus (ALH) at stereotaxic coordinates of 1.5/1.5/8.5 (for animals in Group $\mathrm{S}^{+}$), in the dorsomedial tegmentum (DMT) at the coordinates $-4.0 / 1.0 / 6.0$ (for the $S s$ in Group $S^{-}$), or in the reticular formation (RF) at the coordinates $-3.5 / 2.0 / 8.5$ (for the animals in Group $S^{n}$ ). In describing the coordinates, the three figures represent distances in millimeters from the primary skull marking bregma in the frontal, lateral, and horizontal directions, respectively. In the frontal direction, plus numbers are anterior and minus numbers are posterior in relation to bregma. All implantations were in the right side of the brain.

ICS testing began for all animals after a 4- to 5-day recovery period following surgery. It consisted of a daily $1 / 2-h$ session in the Skinner boxes on 3 successive days. At the beginning of each session, the rats were induced to commence self-stimulation by 10 deliveries of noncontingent ICS. Only one current level was used in the present study ( 150 microamps); it was suggested on the basis of the work of Hodos and Valenstein (1962) that, at one given current level, preference tests and rate measures correlate perfectly.

The animals in Group $\mathrm{S}^{+}$(implanted in ALH) performed self-stimulation. As in an earlier study (St-Laurent \& Beaugrand, 1972), the intensity of self-stimulation was graded in three categories: very high (over 1800 responses for $30 \mathrm{~min}$ ), high (901-1800), and low (150-900). In this experiment the 10 animals in Group $\mathrm{S}^{+}$made a median number of 533 barpresses in the last $1 / 2-h$ session (range 150-2,664). One $S$ was classified as very high, two as high, and seven as low self-stimulators. The Ss in Group $\mathrm{S}^{-}$(implanted in DMT) did not show self-stimulation "approach behavior," but rather they displayed "escape behavior" contingent upon the administration of the ICS. Neither self-stimulation nor escape behavior was observed in the rats used in Group $S^{n}$ when ICS was applied. Seven animals in the latter group were implanted in the RF, while the three remaining ones were implanted in the ALH. These three failed to reach the criterion of low self-stimulators and failed to show approach behavior. In Group $\mathrm{S}^{\mathbf{n}}$, all Ss showing self-stimulation, escape behavior, or any prominent motor activities contingent upon ICS administration were disregarded from this experiment. This qualified the animals as neutral Ss.

The animals in Groups NS and TC were screened, as were the rats in the other groups. These were taken from the groups of animals implanted in the ALH (two in Group NS and one in Group TC), in the DMT (four in Group NS and seven in Group TC), and in the RF (four in Group NS and two in Group TC). For all animals, avoidance training began the day following the screening for the effects of ICS.

For Groups $\mathrm{S}^{+}, \mathrm{S}^{-}, \mathrm{S}^{\mathbf{n}}$, and NS, the avoidance procedure consisted of the following sequence: (1) avoidance acquisition, (2) flooding, and (3) extinction testing, the difference between the four groups being the kind of ICS (positive, aversive, neutral, or none) being applied during flooding. For Group TC, a time control group, neither ICS nor flooding treatment was given.

\section{Avoidance Acquisition}

The avoidance acquisition was the same for all groups. The first trial of avoidance acquisition was begun by the animal being dropped onto the grid floor. After $10 \mathrm{sec}$, a 1.3-microamp shock was passed through the grid, terminating when the animal climbed onto the ledge. The animal remained on the ledge for $30 \mathrm{sec}$, at which point the shelf automatically retracted for a moment, dropping the animal back onto the floor and starting a new 10 -sec warning interval. If during the $30-\mathrm{sec}$ intertrial interval (ITI) the animal fell off the ledge, a new trial was automatically begun. The avoidance response was said to have been learned to criterion when the animal avoided shock (by climbing onto the ledge within $10 \mathrm{sec}$ ) on 10 consecutive triais.

\section{Flooding}

After the tenth consecutive avoidance response, the rats in Groups $\mathrm{S}^{+}, \mathrm{S}^{-}, \mathrm{S}^{\mathrm{n}}$, and NS were removed from the apparatus and placed in a nearby plastic pail for a 1-min waiting period. During this time, the ledge was removed from the avoidance box, and the wire leads from the stimulator were attached to the electrodes. After this $1 \mathrm{~min}$ in the pail, each rat in these four groups was placed on the grid floor of the avoidance apparatus, where it remained for $5 \mathrm{~min}$ of flooding, with each animal thus being forced to remain in the situation which it had learned to fear, namely, on the grid floor. Of course, during this time the shocker (for delivering footshock) was disconnected.

During the 5-min flooding period, Groups $\mathrm{S}^{+}, \mathrm{S}^{-}$, and $\mathrm{S}^{\mathbf{n}}$ received noncontingent rewarding, aversive, or neutral ICS, respectively ( 150 microamps, one 0.25 -sec pulse every $5 \mathrm{sec}$ ), while Group NS received no stimulation. For these four groups, the spontaneous behavior in which the animals engaged during 
Fig. 1. Sections $A$ and $B$ are drawings reproduced from Pellegrino and Cushman (1967). Selected abbreviations: CA, anterior commissure; $\mathbf{C C}$, corpus callosum; $\mathbf{C O}$, optic chiasma; CP, posterior commissure; CPU, caudate nucleus-Putamen; IP, interpeduncular nucleus; $L M$, medial lemniscus; LS, lateral septum nucleus; MFB, medial forebrain bundle; MS, medial septal nucleus; NCP, bed nucleus of the posterior commissure; NOT, nucleus of olfactory tract; NPT, posterior nucleus of thalamus; POA, lateral preoptic area; PVG, central gray substance; RF, reticular formation of mesencephalon; SN, substantia nigra; TOL, lateral olfactory tract; $V$, ventricule. Behavioral phenomena are indicated as follows: $\Delta=$ high self-stimulator, $\bullet=$ low self-stimulator, $0=$ neutral, $\star=$ aversive.
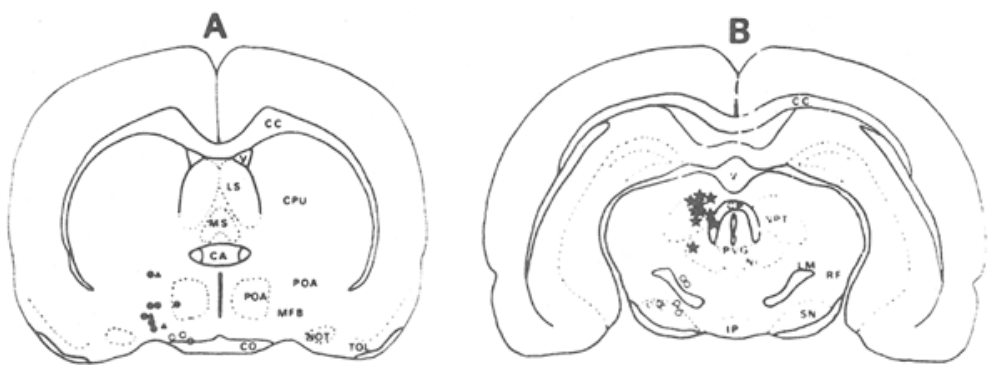

the flooding experience was systematically observed and recorded. The incidence of abortive attempts to avoid, freezing, grooming, and general activity were quantitatively measured by means of a time-sampling technique described in previous papers (Baum, 1969, 1970). After the acquisition period, the animals in Group TC spent an overall $7 \mathrm{~min}$ in a neutral environment (namely, a plastic pail) before they started extinction testing.

\section{Extinction}

After flooding was completed, every rat in Groups $\mathrm{S}^{+}, \mathrm{S}^{-}, \mathrm{S}^{\mathbf{n}}$, and NS was again removed from the avoidance apparatus and placed in a plastic pail for an additional 1-min waiting period. During this minute, the ledge was reinserted and reengaged in the avoidance box, and the stimulator leads were disconnected. At the end of this minute, extinction was carried out in the same manner for all the five groups. To begin extinction, the animal was placed on the ledge to begin a 30 -sec ITI preceding the first extinction trial. Trials were then carried out as during acquisition, with the exception of there being no shock present at any time. When the animal remained on the grid floor for 5 consecutive min without responding, it was said to have extinguished.

After the completion of the avoidance procedure, the animals in Groups $\mathrm{S}^{+}, \mathrm{S}^{-}$, and $\mathrm{S}^{n}$ were sacrificed and perfused with $10 \%$ formalin solution. Brain sections were stained with the Luxol fast blue technique of Kluver-Barrera for histological verification of electrode positions.

\section{RESULTS}

\section{Histological Findings}

The various electrode placements are illustrated in Fig. 1. In the Group $\mathrm{S}^{+}$animals, the probes were located in the lateral preoptic area in the anterior part of the MFB. In the Group S- rats, the probes fell in an area lateral and dorsal to the central gray substance, more specifically in the posterior commissure, in the bed nucleus of the posterior commissure, and in the posterior nucleus of thalamus. In the Group $\mathrm{Sn}^{\mathrm{n}}$ animals, the probes were placed anteriorly in an area bordering the optic chiasma under the medial preoptic area, posteriorly at the level of the mesencephalon above and under the medial lemniscus, or in the dorsolateral part of the substantia nigra.

\section{Behavioral Findings}

The results are summarized in Table 1. No significant difference was observed between the five groups in the number of shocks received in acquisition $[\mathrm{H}=3.52$, n.s., Kruskal-Wallis nonparametric statistical analysis (Siegel, 1956)]. The number of trials required by each group in order to reach the criterion of avoidance learning in acquisition was found to be not significantly different $(\mathrm{H}=3.53$, n.s. $)$ In extinction testing, the number of trials made by each group before reaching the extinction criterion did not differ significantly $(\mathrm{H}=4.07$, n.s.).

During flooding, the animals in Group $\mathrm{S}^{-}$engaged significantly more in freezing behavior and less in abortive avoidance when compared with Group $\mathrm{S}^{+}, \mathrm{S}^{n}$, or NS. However, the incidence of grooming and general activity displayed during flooding was very low in all groups and these behavioral measures did not differ significantly from one group to another.

Table 1

Summary of the Acquisition and Extinction of the Avoidance Response for the Five Groups

\begin{tabular}{|c|c|c|c|c|c|c|}
\hline & $\begin{array}{c}\text { Group TC } \\
(\mathrm{N}=10)\end{array}$ & $\begin{array}{c}\text { Group NS } \\
(\mathrm{N}=10)\end{array}$ & $\begin{array}{r}\text { Group } S+ \\
(\mathrm{N}=10)\end{array}$ & $\begin{array}{c}\text { Group } S- \\
(\mathrm{N}=10)\end{array}$ & $\begin{array}{c}\text { Group } S^{n} \\
(\mathrm{~N}=10)\end{array}$ & $\begin{array}{c}\text { Kruskal- } \\
\text { Wallis } \mathrm{H}=\end{array}$ \\
\hline Median number of shocks received in acquisttion & 4.5 & 4.5 & 6.0 & 4.5 & 6.0 & $3.52 *$ \\
\hline Median number of trials to criterion & 20.5 & 17.0 & 22.5 & 19.5 & 22.5 & $3.53 *$ \\
\hline Median number of responses in extinction & 62.5 & 34.5 & 42.0 & 12.0 & 31.5 & $4.07 \dagger$ \\
\hline
\end{tabular}

*not significant $\quad t p>.1$, not significant 


\section{DISCUSSION}

The results of this experiment showed no facilitative effect on avoidance extinction produced by positive, negative, neutral, or no brain stimulation during flooding. In fact, the number of trials made in extinction testing in each group was high and did not differ significantly from one group to another.

In comparison with our previous study (Baum; Leclerc, \& St-Laurent, in press) the results of the present experiment suggest that the effects of the stimulation of anterior structures, namely, the anterior lateral hypothalamus (ALH) and dorsomedial tegmentum (DMT), during flooding differ from the effects of the stimulation of posterior structures, namely, the posterior lateral hypothalamus (PLH) and reticularis pontis caudalis (RPC).

During flooding, the ICS of anterior areas of the brain seems to induce less-intense approach and escape behaviors than the same behaviors induced by ICS in posterior areas. The animals stimulated in the ALH during flooding displayed only mild approach behavior, while the rats implanted in the PLH showed intense approach behavior (i.e., exploration, increase in motor activities, and sniffing). In addition, freezing was the dominant behavior in Ss implanted in the DMT, while abortive attempts to avoid were observed in the animal Ss implanted in the RPC.

These different effects on the behaviors induced by ICS during flooding correlate with the different results observed in avoidance extinction in both experiments. The number of trials made in extinction was significantly reduced by the application of ICS in PLH during flooding (Gordon \& Baum, 1971; Baum, Leclerc, \& St-Laurent, in press). No effects on this behavioral measure were found when ICS in ALH was applied. On the other hand, when compared to the Group PLH, Group RPC was found to perform more responses in extinction, while there was no significant difference in extinction testing between Group RPC and a time control group which did not receive any stimulation during flooding (Baum, Leclerc, \& St-Laurent, in press). However, Group DMT in the present study did not differ significantly from any other group in resistance to extinction, including the ALH group. Thus, the behavioral differences observed during flooding and avoidance extinction between the present experiment and our earlier study suggest that ICS at a rate of one stimulation each $5 \mathrm{sec}$ in the ALH and DMT had fewer induced approach-behavior and/or counterconditioning properties and fewer aversive effects, respectively, than ICS at the same rate in the PLH and RPC. These latter two brain areas induced approach and escape behaviors, respectively.

It may be suggested that the rate of ICS during flooding, independent of the structures stimulated, is an important factor in the outcome of flooding action on the extinction of a previously learned response. In other words, increasing the rate of ICS from one per $5 \mathrm{sec}$ to one per $2 \mathrm{sec}$ or even one per second, may induce exploration (approach behavior) and abortive attempts to avoid (escape behavior) in Ss implanted in the ALH and DMT, respectively, in a way comparable to the same behaviors induced by ICS at a lower rate (one stimulation per $5 \mathrm{sec}$ ) in the PLH and RPC.

In addition, the duration of the ICS testing period $(1 / 2 \mathrm{~h})$ for SS may be considered as being too brief in order to establish the rewarding properties of ICS in Group ALH. The previous statement is suggested on the basis that 7 out of 10 self-stimulators were classified as low self-stimulators, while only 2 were high and 1 was very high. Again in comparison with our previous study, the ICS testing period was longer ( $1 \mathrm{~h}$ in duration) and most of the self-stimulators $(\mathrm{N}=8)$ were either high or very high; only one was low.

At any rate, the present experiment showed that in rats the stimulation of the ALH and DMT structures during flooding does not enhance the facilitative action of flooding on avoidance extinction, this observation differing from the one made in our earlier experiment (Baum, Leclerc, \& St-Laurent, in press) dealing with the stimulation of the PLH and RPC structures during flooding. Moreover, the behavioral observations made during flooding in this experiment, in comparison with our previous study (Baum et al, in press), give more support to the suggestion made by St-Laurent and Beaugrand (1972) that a gradient of intensity exists in the behaviors induced by stimulation of brain structures along the MFB and more dorsal pathways, the stimulation of posterior areas being more reinforcing (positively and negatively) than the stimulation of anterior structures.

\section{REFERENCES}

Baum, M. Rapid extinction of an avoidance response following a period of response prevention in the avoidance apparatus. Psychological Reports, 1966, 18, 59-64.

Baum, M. Extinction of an avoidance response motivated by intense fear: Social facilitation of the action of response prevention (flooding) in rats. Behaviour Research \& Therapy, $1969,7,57-62$.

Baum, M. Extinction of avoidance responding through response prevention (flooding). Psychological Bulletin, 1970, 74, 276-284.

Baum, M., Leclerc, R., \& St-Laurent, J. Rewarding vs. aversive intercranial stimulation administered during flooding (response prevention) in rats. Psychological Reports, in press. Gordon, A., \& Baum, M. Increased efficacy of flooding (response prevention) in rats through positive intracranial stimulation. Journal of Comparative \& Physiological Psychology, 1971, 75, 68-72.

Hodos, W., \& Valenstein, E. S. An evaluation of response rate as a measure of rewarding intracranial stimulation. Journal of Comparative \& Physiological Psychology, 1962, 55, 80-84.

Hogan, R. A., \& Kirchner, J. H. Preliminary rennrt of the 
extinction of learned fears via short-term implosive therapy. Journal of Abnormal Psychology, 1967, 72, 106-109.

Kirchner, J. H., \& Hogan, R. A. The therapist variable in the implosion of phobias. Psychotherapy: Theory, Research \& Practice, 1966, 3, 102-104.

Lederhendler, I., \& Baum, M. Mechanical facilitation of the action of response prevention (flooding) in rats. Behaviour Research \& Therapy, 1970, 8, 43-48.

Olds, J. Studies of neuropharmacologicals by electrical and chemical manipulation of the brain in animals with chronically implanted electrodes. In P. B. Bradley et al (Eds.), Neuropsychopharmacology Proceedings of the First International Congress of Neuropharmacology, Rome, September 1958, 20-32.

Olds, J., \& Milner, P. Positive reinforcement produced by electrical stimulation of septal area and other regions of rat brain. Journal of Comparative \& Physiological Psychology, $1954,47,419-427$.

Pellegrino, L. J., \& Cushman, A. J. A stereotaxic atlas of the rat brain. New York: Appleton-Century-Crofts, 1967.

St-Laurent, J., \& Beaugrand, J. Brain stimulation, reinforcement, and behavior. Revue Canadienne de Biologie, 1972, 31 (supplement), 193-213.

Siegel, S. Nonparametric statistics for the behavioral sciences. New York: McGraw-Hill, 1956.

(Received for publication August 14, 1972; accepted October 2, 1972.) 